\title{
COMPLETE REFINEMENTS OF THE BEREZIN NUMBER INEQUALITIES
}

\author{
Mojtaba BAKHERAD, RaHmatollah LASHKARIPOUR, MONiRE \\ HAJMOHAMADI AND ULAS YAMANCI
}

Abstract. In this paper, several refinements of the Berezin number inequalities are obtained. We generalize inequalities involving powers of the Berezin number for product of two operators acting on a reproducing kernel Hilbert space $\mathscr{H}=\mathscr{H}(\Omega)$ and also improve them. Among other inequalities, it is shown that if $A, B \in \mathscr{B}(\mathscr{H})$ such that $|A| B=B^{*}|A|, f$ and $g$ are nonnegative continuous functions on $[0, \infty)$ satisfying $f(t) g(t)=t(t \geqslant 0)$, then

$\operatorname{ber}^{p}(A B) \leqslant r^{p}(B) \times$

$$
\left(\operatorname{ber}\left(\frac{1}{\alpha} f^{\alpha p}(|A|)+\frac{1}{\beta} g^{\beta p}\left(\left|A^{*}\right|\right)\right)-r_{0}\left(\left\langle f^{2}(|A|) \hat{k}_{\lambda}, \hat{k}_{\lambda}\right\rangle^{\alpha p / 4}-\left\langle g^{2}\left(\left|A^{*}\right|\right) \hat{k}_{\lambda}, \hat{k}_{\lambda}\right\rangle^{\beta p / 4}\right)^{2}\right)
$$

for every $p \geqslant 1, \alpha \geqslant \beta>1$ with $\frac{1}{\alpha}+\frac{1}{\beta}=1, \beta p \geqslant 2$ and $r_{0}=\min \left\{\frac{1}{\alpha}, \frac{1}{\beta}\right\}$.

Mathematics subject classification (2010): Primary 47A30, Secondary 15A60, 30E20, 47A12. Keywords and phrases: Berezin number; Berezin symbol; Heinz means.

\section{REFERENCES}

[1] M. W. Alomari, Numerical radius inequalities for Hilbert space operators, Math. USSR-Izv. ArXive: $1810.05710 \mathrm{v} 2$.

[2] M. BAKHERAD, Some Berezin number inequalities for operator matrices, Czechoslovak Math. J. 68 (4) (2018), 997-1009.

[3] M. BAKherad, M.T. KARAev, Berezin number inequalities for operators, Concr. Oper. 6 (2019), no. $1,33-43$.

[4] F. A. BEREZIN, Covariant and contravariant symbols for operators, Math. USSR-Izv. 6 (1972), 1117 1151.

[5] F. A. BerezIn, Quantizations, Math. USSR-Izv. 8 (1974), 1109-1163.

[6] H. Bohr, A theorem concerning power series, Proc. Lond. Math. Soc., 2(13) (1914), 1-5.

[7] M. T. GARAYEV, M. GÜRDAL, A. OKUDAN, Hardy-Hilbert's inequality and a power inequality for Berezin numbers for operators, Math. Inequal. Appl. 3 (2016), 883-891.

[8] M. T. Garayev, M. GÜrdal, S. Saltan, Hardy type inequaltiy for reproducing kernel Hilbert space operators and related problems, Positivity 21 (2017), 1615-1623.

[9] M. Hajmohamadi, R. LAShkaripour, M. BAKHerad, Some generalizations of numerical radius on off-diagonal part of $2 \times 2$ operator matrices, J. Math. Inequal. 12 (2) (2018), 447-457.

[10] M. Hajmohamadi, R. Lashkaripour, M. Bakherad, Improvements of Berezin number inequalities, Linear and Multilinear Algebra, https://doi.org/10.1080/03081087.2018.1538310 (to appear).

[11] M. Hajmohamadi, R. Lashkaripour, M. Bakherad, Further refinements of generalized numerical radius inequalities for Hilbert space operators, Georgian Math. J. https://doi.org/10.1515/gmj-2019-2023 (to appear).

[12] P. R. Halmos, A Hilbert Space Problem Book, 2nd ed., springer, New York, 1982.

[13] M. T. KARAEV, Berezin symbol and invertibility of operators on the functional Hilbert spaces, J. Funct. Anal. 238 (2006), 181-192. 
[14] M. T. Karaev, Functional analysis proofs of Abels theorems, Proc. Amer. Math. Soc. 132 (2004), 2327-2329.

[15] M. T. Karaev, S. Saltan, Some results on Berezin symbols, Complex Var. Theory Appl. 50 (3) (2005), 185-193.

[16] T. Kosem, Inequalities between $\|f(A+B)\|$ and $\|f(A)+f(B)\|$, Linear Algebra Appl. 418 (1) (2006), 153-160.

[17] F. Kittaneh, Notes on some inequalities for Hilbert space operators, Publ. Res. Inst. Math. Sci. 24 (1988), 283-293.

[18] F. Kittaneh, Spectral radius inequalities for Hilbert space operators, Proc. Amer. Math. Soc., 134 (2) (2005), 385-390.

[19] F. Kittaneh, Y. Manasrah, Improved Young and Heinz inequalities for matrices, J. Math. Anal. Appl. 361 (2010), 262-269.

[20] C. A. MCCARThy, $C_{p}$, Israel J. Math. 5 (1967), 249-271.

[21] E. Nordgren, P. Rosenthal, Boundary values of Berezin symbols, Oper. Theory Adv. Appl. 73 (1994), 362-368.

[22] U. Yamanci, M. GÜrdal, M. T. Garayev, Berezin number inequality for convex function in reproducing kernel Hilbert space, Filomat, 31 (2017), 5711-5717.

[23] U. YAMANCI M. GÜRDAL, On numerical radius and Berezin number inequalities for reproducing kernel Hilbert space, New York J. Math. 23 (2017), 1531-1537.

[24] U. Yamanci, M. T. Garayev, C. Çelik, Hardy-Hilbert type inequality in reproducing kernel Hilbert space: its applications and related results, Linear and Multilinear Algebra 67 (4) (2019), 830-842. 